\title{
Cardiorespiratory Coupling in Asthmatic Children
}

\author{
Javier Milagro ${ }^{1,2}$, Javier Gracia ${ }^{3}$, Ville-Pekka Sepp $\ddot{A}^{4}$, Jussi Karjalainen ${ }^{5}$, Marita Paassilta ${ }^{5}$, \\ Michele Orini ${ }^{6}$, Eduardo Gil ${ }^{1,2}$, Raquel Bailón ${ }^{1,2}$, Jari Viik ${ }^{3}$ \\ ${ }^{1}$ BSICoS Group, I3A, IIS Aragón, University of Zaragoza, Zaragoza, Spain \\ ${ }^{2}$ CIBER - Biengineering, Biomaterials and Nanomedicine (CIBER-BBN), Madrid, Spain \\ ${ }^{3}$ Faculty of Medicine and Health Technology, Tampere University, Tampere, Finland \\ ${ }^{4}$ Revenio Research Ltd., Vantaa, Finland \\ ${ }^{5}$ Allergy Center of the Tampere University Hospital, Tampere, Finland \\ ${ }^{6}$ Institute of Cardiovascular Science, University College of London, United Kingdom
}

\begin{abstract}
The relationship between cardiac and respiratory autonomic control has been suggested to be altered in several respiratory disorders. However, and despite the increasing prevalence of asthma, there are not studies assessing the cardiorespiratory coupling (CRC) with respect to the asthmatic status. Since altered autonomic control has been suggested to play a major role in asthma, in this work overnight CRC was assessed in a group of 67 children who underwent a three-month inhaled corticosteroids treatment. After treatment completion, CRC was reduced $(p<0.005)$ in the subjects without or with a low risk of asthma, whereas it kept unchanged in those with a worse prognosis, suggesting that an altered interaction between cardiac and respiratory activity might be related with an increased risk of asthma.
\end{abstract}

\section{Introduction}

Asthma is a chronic respiratory disorder affecting people of any age, although it is prone to start during childhood [1]. Despite its increasing prevalence [2], the underlying physiological mechanisms originating asthma are not completely understood. Moreover, its diagnosis remains compromised in young children, who are unable to perform repeatable spirometric maneuvers [3].

Asthma is characterized by variable broncho-constriction and airway hyper-reactivity. Since bronchomotor tone and broncho-constriction are driven by the vagal branch of the autonomic nervous system (ANS), altered autonomic control has been proposed to play a major role in the pathogenesis of asthma. This hypothesis has motivated large research, and whereas excessive vagally-mediated bronchoconstriction is a well-know symptom in asthmatics, an exaggerated cardiac vagal activity in response to autonomic tests has been also suggested in children [4] and adults [5]. However, to the best of our knowledge, the relationship between cardiac and respiratory activity has not been studied with respect to asthmatic condition. Since cardiorespiratory coupling (CRC) has been suggested to be altered in some respiratory disorders [6], in this work we assessed the CRC in a group of children classified attending to their asthmatic condition, under and after inhaled corticosteroids (ICS) treatment. If CRC differed among groups, noninvasive CRC assessment could represent a novel approach for studying asthma in young children.

\section{Materials and Methods}

\subsection{Database}

67 children aged 2.5 (0.9-5.7) years old (median and range) who were prescribed three-month ICS treatment for obstructive bronchitis were enrolled. Electrocardiographic (ECG) and impedance pneumography (IP) signals were acquired from each subject during three nights (whole night recordings) at different stages with respect to treatment completion. The first recording (R1) was performed 1 week before concluding the treatment, whereas the second (R2) and third (R3) recordings were conducted 1-2 weeks and 3-4 weeks after treatment completion. All the recordings were performed at each subject home with a custom designed recording device developed at Tampere University [7]. The parents of the subjects were conveniently instructed on the use of the device, and they were also requested to annotate the sleep onset and offset of the children. After a six-month follow up, a pediatric pulmonologist classified the subjects into three groups attending to their current asthma (CA) status, so that they were labeled as having current asthma (CA-Y, 35 subjects) if any medication for the control of asthma was prescribed in the 
follow up period due to wheezing, cough or shortness of breath reversible with bronchodilators. On the other hand, those subjects who did not fulfilled the previous criteria but were prescribed with short-term medication for asthma were classified as possible current asthma (CA-P, 16 subjects), whereas the remaining children were labeled as not having current asthma (CA-N, 16 subjects). None of the subjects had previous history of other respiratory diseases that might interfere with asthma or with ECG and IP signals acquisition. Written informed consent was received from the parents of all the children. The study was approved by the local Ethics Committees in accordance with the Declaration of Helsinki.

\subsection{Signal Preprocessing}

Baseline wander was removed from the ECGs, and respiratory signals were filtered with a $3^{\text {rd }}$-order Butterworth band-pass filter (0.05-0.5 Hz cut-off frequencies). Then, beat time occurrences were detected using the waveletbased approach in [8], and ectopic beat detection was performed following the same methodology proposed in [9]. Afterwards, the time-varying integral pulse frequency modulation (TVIPFM) model [10] was used for estimating the modulating signal, $m(t)$, which carries cardiac autonomic modulation information. First, an instantaneous HR signal, $d_{\mathrm{HR}}(t)$, was derived from the beat time occurrences series, also accounting for the presence of ectopic beats. A low-pass filtered version of $d_{\mathrm{HR}}(t), d_{\mathrm{HRM}}(t)$, was obtained to account for the time-varying mean HR, and the modulating signal was estimated as $m(t)=\left(d_{\mathrm{HR}}(t)-d_{\mathrm{HRM}}(t)\right) / d_{\mathrm{HRM}}(t)$. On the other hand, the instantaneous respiratory rate, $F_{\mathrm{r}}$, was estimated for each subject from its IP signal, as proposed in [11]. The modulating, IP and instantaneous respiratory rate signals were resampled at $4 \mathrm{~Hz}$. Signals were only analyzed during sleeping time.

\subsection{Time-Frequency Coherence Analysis}

The complex analytical signal representation of the modulating and the IP signals, $x_{\mathrm{HRV}}(t)$ and $x_{\mathrm{IP}}(t)$ respectively, were obtained using the Hilbert transform. Afterwards, their time-frequency (TF) cross-spectrum, $\hat{S}_{\mathrm{HRV}, \mathrm{IP}}(t, f)$, was estimated using a TF distribution belonging to Cohen's class, which is defined as:

$$
\hat{S}_{\mathrm{HRV}, \mathrm{IP}}(t, f)=\iint_{-\infty}^{\infty} A_{\mathrm{HRV}, \mathrm{IP}}(\nu, \tau) \Phi(\nu, \tau) e^{j 2 \pi(t \nu-f \tau)} d \nu d \tau
$$

where $A_{\mathrm{HRV}, \mathrm{IP}}(\nu, \tau)$ is the ambiguity function of $x_{\mathrm{HRV}}(t)$ and $x_{\mathrm{IP}}(t)$, and $\Phi(\nu, \tau)$ is a smoothing function in the ambiguity domain (in this work it was selected as an elliptic exponential kernel). They are respectively defined as:

$$
\begin{gathered}
A_{\mathrm{HRV}, \mathrm{IP}}(\nu, \tau)=\int_{-\infty}^{\infty} x_{\mathrm{HRV}}\left(t+\frac{\tau}{2}\right) x_{\mathrm{IP}}^{*}\left(t-\frac{\tau}{2}\right) e^{-j 2 \pi \nu t} d t \\
\Phi(\nu, \tau)=e^{-\pi\left[\left(\frac{\nu}{\nu_{0}}\right)^{2}+\left(\frac{\tau}{\tau_{0}}\right)^{2}\right]^{2 \lambda}}
\end{gathered}
$$

The smoothing kernel in Eq. 2 allows to adjust the time and frequency resolution independently by modifying its shape through the parameters $\nu_{0}$ and $\tau_{0}$, respectively. On the other hand, its roll-off factor is controlled by $\lambda$. In this work, these parameters were fixed to $\nu_{0}=0.045, \tau_{0}=0.05$ and $\lambda=0.3$. Afterwards, the TF coherence (TFC) distribution was obtained as:

$$
\hat{\gamma}^{2}(t, f)=\frac{\left|\hat{S}_{\mathrm{HRV}, \mathrm{IP}}(t, f)\right|^{2}}{\hat{S}_{\mathrm{HRV}}(t, f) \hat{S}_{\mathrm{IP}}(t, f)},
$$

where $\hat{S}_{\mathrm{HRV}}(t, f)$ and $\hat{S}_{\mathrm{IP}}(t, f)$ are the TF spectra of the modulating and the IP signals estimated as in Eq. 1. CRC was then measured using two different parameters. The first one consists in the bandwidth for each time instant $t, \Omega(t)$, in which both spectra are considered coherent, i.e., the bandwidth for which $\hat{\gamma}^{2}(t, f) \geq \gamma_{\mathrm{TH}}(t, f ; \alpha)$, being $\gamma_{\mathrm{TH}}(t, f ; \alpha)$ the signal-independent threshold defined in [12], with $\alpha=0.01$. An example of the calculation of $\Omega$ is displayed in Fig. 1. On the other hand, the mean coherence in $\Omega(t), \hat{\gamma}_{\Omega}^{2}(t)$, was calculated as:

$$
\hat{\gamma}_{\Omega}^{2}(t)=\frac{1}{\Omega(t)} \int_{\Omega_{1}(t)}^{\Omega_{2}(t)} \hat{\gamma}^{2}(t, f) d f,
$$

where $\Omega_{1}(t)$ and $\Omega_{2}(t)$ represent the onset and offset of the frequency band for which $\hat{\gamma}^{2}(t, f) \geq \gamma_{\mathrm{TH}}(t, f ; \alpha)$ in the time instant $t$.

\subsection{Statistical Analysis}

The median of each of the proposed parameters was calculated in two-hour windows with one-hour overlap, so that at least one complete sleep cycle was covered [13]. This process was repeated for each subject and recording session. Normality of the data was rejected using a Kolmogorov-Smirnov test. Afterwards, inter-day differences were assessed in each group by comparing the results of each two-hour interval, using paired Wilcoxon signed-rank tests, whereas inter-group differences were assessed through a rank-sum Wilcoxon test. Bonferroni correction was applied, so the significance threshold for considering statistical differences was set to 0.017 .

\section{Results}

A general decrease in $\Omega$ and $\hat{\gamma}_{\Omega}^{2}$ was assessed in R3 with respect to $\mathrm{R} 1$ and $\mathrm{R} 2$ in the $\mathrm{CA}-\mathrm{N}$ group. However, these 


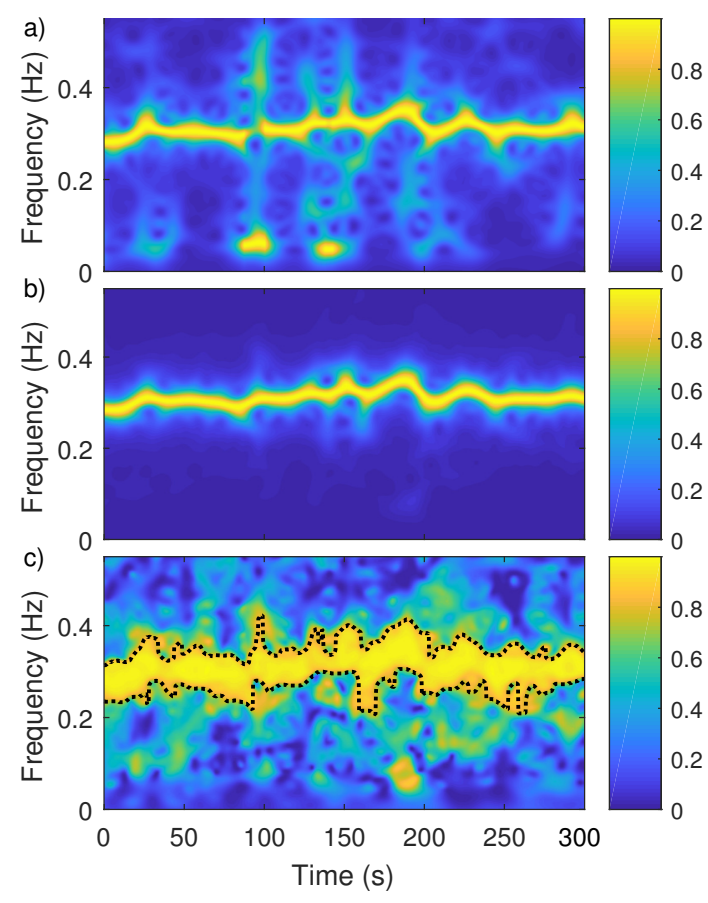

Figure 1. A five-minute segment of the normalized timefrequency distribution of the heart rate modulating signal (a)) and the impedance pneumography signal (b)) are displayed. In c), the time-frequency coherence distribution is depicted. The black dotted lines represent the limits between which $\hat{\gamma}^{2}(t, f) \geq \gamma_{\mathrm{TH}}(t, f ; \alpha)$ (see text for details).

Table 1. Median of the medians (interquartile range) of the proposed parameters in a two-hour window centered at 2 a.m., for each recording day. Significant differences $(\mathrm{p}<0.005)$ between R3 and R2 are indicated with *.

\begin{tabular}{ccccc}
\hline \hline & & CA-N $(\mathrm{N}=16)$ & CA-P $(\mathrm{N}=16)$ & CA-Y $(\mathrm{N}=35)$ \\
\hline \multirow{3}{*}{$\Omega(\mathrm{Hz})$} & $\mathrm{R} 1$ & $0.089(0.028)$ & $0.097(0.029)$ & $0.088(0.027)$ \\
& $\mathrm{R} 2$ & $0.089(0.041)$ & $0.087(0.033)$ & $0.091(0.025)$ \\
& $\mathrm{R} 3$ & $0.079(0.021)^{*}$ & $0.096(0.021)$ & $0.089(0.027)$ \\
\hline \multirow{3}{*}{$\hat{\gamma}_{\Omega}^{2}$ (n.u) } & R1 & $0.914(0.015)$ & $0.915(0.011)$ & $0.912(0.012)$ \\
& R2 & $0.913(0.014)$ & $0.912(0.008)$ & $0.914(0.008)$ \\
& R3 & $0.908(0.011)^{*}$ & $0.916(0.010)$ & $0.913(0.011)$ \\
\hline \hline
\end{tabular}

differences only turned statistically significant when comparing R3 and R2. In this case, $\hat{\gamma}_{\Omega}^{2}$ significantly reduced from 01 to 03 a.m., whereas $\Omega$ was lower from 01 to 04 a.m. No differences were found for CA-P or CA-Y at any time, nor among groups in any of the recording days. These results are summarized in Fig. 2 and Table 1.

\section{Discussion}

All the children underwent the same three-month ICS treatment. However, several weeks after treatment completion, CRC was reduced in the group without current asthma, whereas it kept unchanged in the children with a worse prognosis. Since CRC is related to how the respiratory activity modulates the heart rate, an increased CRC would be reflecting a more synchronous vagal modulation of respiratory and cardiac rhythms. This hypothesis is in agreement with the concept of illness as a state of reduced complexity [14], and suggests that heart rate variability (HRV) might be more dependent on the respiratory activity in asthma or in subjects at an increased risk of developing asthma in the future. However, the nature of this behavior remains unclear. A possible explanation would be a higher interaction between the cardiac and respiratory centers at the brainstem level in asthmatics. On the other hand, Fryer et al. suggested that $\mathrm{M}_{2}$ muscarinic receptors dysfunction could result in an excessive vagal activity, causing airway hyper-responsiveness [15]. If cardiac vagal fibers also presented this dysfunction, the simultaneous reduction in the adaptability of cardiac and respiratory vagal control due to excessive acetylcholine release could resemble an increased CRC. Nevertheless, further research is needed in order to understand the neural control in asthma.

CRC was assessed using two parameters extracted from the TFC distribution. The use of the TF distribution in Eq. 1 was motivated by its high resolution, and the possibility to control the degree of time and frequency filtering independently [12]. Whereas statistical differences were only found in R3 with respect to R2, also a strong tendency with respect to R1 was assessed. The fact that both $\Omega$ and $\hat{\gamma}_{\Omega}^{2}$ decreased after treatment in the CA-N group suggests a reduction in the frequency span in which HRV is governed by respiration, but also in the strength of the interdependence of cardiac and respiratory control. Although subject characterization was performed by calculating the median of each parameter in two-hour windows in order to minimize the effect of sleep stages [13], the absence of polysomnographic recordings constitutes a limitation.

Although the physiological mechanisms underlying asthma are not yet completely understood, past and ongoing research have provided evidence that altered autonomic control could be an important factor. In this way, noninvasive ANS assessment using cardiorespiratory signals analysis could shed some light on the pathogenesis of asthma, and be a potential tool for its monitoring, which remains a challenge in the case of young children.

\section{Conclusion}

Cardiorespiratory coupling was reduced after a period of inhaled corticosteroids treatment in a group of children without or at low risk of asthma, whereas it kept unchanged in children with worse prognosis. These results suggest that vagal modulation of respiratory and heart rhythms might be more synchronized in asthma, and that cardiorespiratory coupling assessment could be a novel approach for 


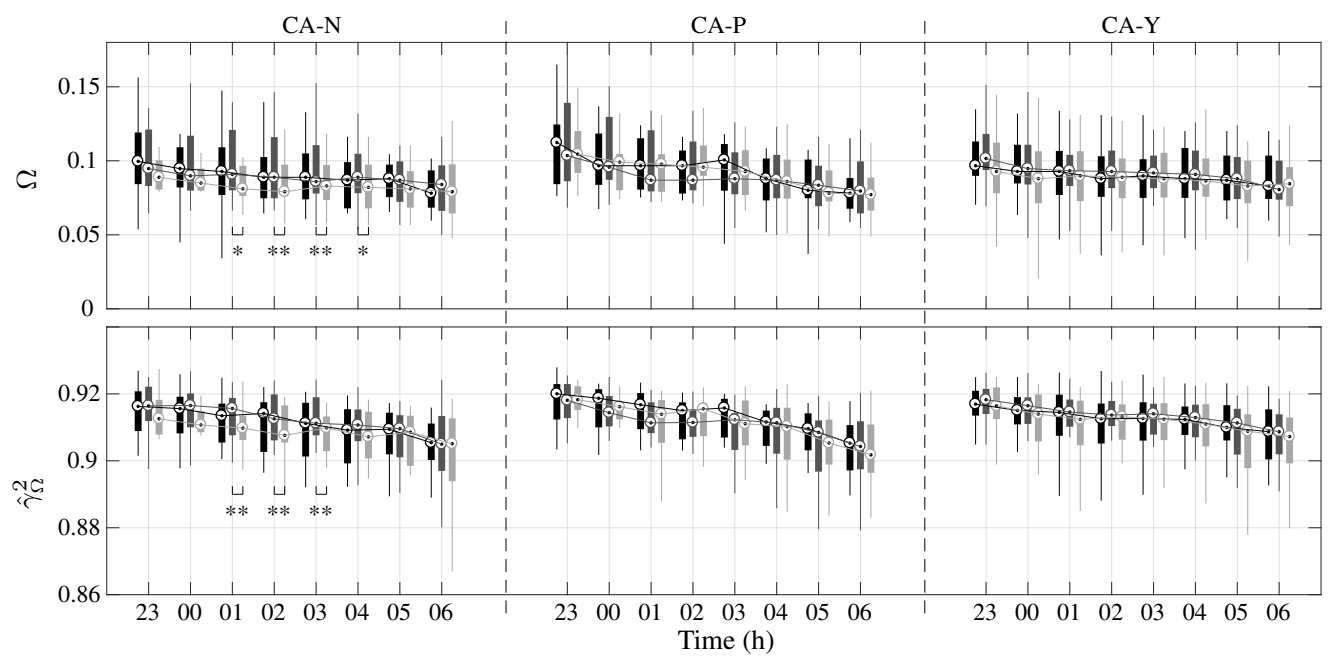

Figure 2. Boxplots of the analyzed parameters in R1 (black), R2 (dark gray) and R3 (light gray) attending to the current asthma status. Each box corresponds to a two-hour window centered in the hour depicted in the figure (although boxes with the same time reference are depicted separately for clarity, same central hour was considered in the analysis). Statistical significant differences are labeled with $*(\mathrm{p} \leq 0.017)$ or $* *(\mathrm{p} \leq 0.005)$.

further research aiming to understand the pathophysiology of this disease.

\section{Acknowledgements}

This work was supported by grant BES-2015-073694 and project RTI2018-097723-B-I00 from Ministerio de Economía y Competitividad, by Gobierno de Aragón (Reference Group BSICoS T39-17R) cofunded by FEDER 2014-2020 "Building Europe from Aragon", by CIBERBBN through Instituto de Salud Carlos III and by Tampere Tuberculosis Foundation. The computation was performed by the ICTS NANBIOSIS, specifically by the High Performance Computing Unit of CIBER-BBN at University of Zaragoza.

\section{References}

[1] Martinez F. Recognizing early asthma. Allergy 1999; 54:24-28.

[2] Lundbäck B, et al. Is asthma prevalence still increasing? Expert Rev Respir Med 2016;10(1):39-51.

[3] Global initiative for asthma. global strategy for asthma management and prevention, 2018. URL https://ginasthma.org/.

[4] Emin O, et al. Autonomic nervous system dysfunction and their relationship with disease severity in children with atopic asthma. Respir Physiol Neurobiol 2012;183(3):206210.

[5] Kallenbach J, et al. Reflex heart rate control in asthma: evidence of parasympathetic overactivity. Chest 1985; 87(5):644-648.

[6] Garcia III AJ, et al. Cardiorespiratory coupling in health and disease. Auton Neurosci 2013;175(1-2):26-37.
[7] Seppä VP, et al. Tidal breathing flow measurement in awake young children by using impedance pneumography. J Appl Physiol 2013;115(11):1725-31.

[8] Martínez JP, et al. A wavelet-based ecg delineator: evaluation on standard databases. IEEE Trans Biomed Eng 2004; 51(4):570-581.

[9] Milagro J, et al. Nocturnal heart rate variability spectrum characterization in preschool children with asthmatic symptoms. IEEE J Biomed Health Inform 2018;22(5):13321340.

[10] Bailón R, et al. The integral pulse frequency modulation model with time-varying threshold: application to heart rate variability analysis during exercise stress testing. IEEE Trans Biomed Eng 2011;58(3):642-652.

[11] Bailón R, et al. A robust method for ecg-based estimation of the respiratory frequency during stress testing. IEEE Trans Biomed Eng 2006;53(7):1273-1285.

[12] Orini M, et al. Characterization of dynamic interactions between cardiovascular signals by time-frequency coherence. IEEE Trans Biomed Eng 2012;59(3):663-673.

[13] Feinberg I. Changes in sleep cycle patterns with age. J Psychiatric Res 1974;10(3-4):283-306.

[14] Goldberger AL. Fractal variability versus pathologic periodicity: complexity loss and stereotypy in disease. Perspect Biol Med 1997;40(4):543-561.

[15] Fryer AD, Jacoby DB. Muscarinic receptors and control of airway smooth muscle. Am J Resp Crit Care Med 1998; 158(supplement_2):S154-S160.

Address for correspondence:

Javier Milagro

Dep. Ingeniería Electrónica y Comunicaciones. Universidad de Zaragoza, C/ María de Luna 1, L.3.07, 50018 Zaragoza, Spain milagro@unizar.es 